\title{
Association Between Stressful Life Events and Female Primary Sjogren's Syndrome and Their Role in Disease Activity: A Retrospective Case- Control Study in China
}

This article was published in the following Dove Press journal:

Neuropsychiatric Disease and Treatment

\author{
FanYan Meng' \\ Shuang Ren' \\ Yun Meng' \\ Ning Tao ${ }^{1,2}$ \\ Jie Zhang'
}

'Traditional Chinese Medicine Department, The First Affiliated Hospital of China Medical University, Shenyang I I000I, People's Republic of China:

${ }^{2}$ Clinical Medicine of Traditional Chinese and Western Medicine, China Medical University, Shenyang II0I22, People's Republic of China
Correspondence: Jie Zhang

Traditional Chinese Medicine Department, The First Affiliated Hospital of China Medical University, I55 Nanjing North Street, Heping District, Shenyang City, Liaoning Province, People's Republic of China Tel +86-24-83283687

Email zhangie945@outlook.com
Purpose: Previous evidence has shown that psychological stress can trigger the onset of autoimmune disease. This study aimed to investigate the prevalence of stressful life events preceding the onset of symptoms in female primary Sjogren's syndrome (pSS) in China compared to controls and the possible associations of stressful life events with disease activity.

Patients and Methods: This was a hospital-based retrospective case-control study. Sixtyseven consecutive female pSS patients and an equal number of age-matched ( \pm 3 years) healthy controls were recruited for assessment using the related Stressful Life Events Scale. The pSS disease activity was measured by the EULAR Sjogren's syndrome disease activity index. Data were analyzed by SPSS, version 23, using chi-square, univariate logistic regression, multiple logistic regression, and partial correlation.

Results: A higher number of negative stressful life events before disease onset in pSS patients compared with healthy controls suggest that these play a role in increasing the risk of disease occurrence $(\mathrm{OR}=2.59,95 \% \mathrm{CI}: 1.87-3.58, p<0.05)$. The number of positive life events did not differ between the two groups. Both the number and severity of the negative stressful life events were also significantly correlated with disease activity.

Conclusion: Patients with pSS experienced more negative stressful life events in the year preceding the onset of symptoms than controls. Negative stressful life events before symptom onset may be risk factors for pSS and may affect pSS disease activity.

Keywords: primary Sjogren's syndrome, stressful life events, retrospective case-control study, risk factors

\section{Introduction}

Primary Sjogren's Syndrome (pSS) is a chronic autoimmune disease characterized by the infiltration of lymphocytes cells in the salivary and lacrimal glands, resulting in the destruction of the parenchymal tissue, thus often leading to severe dry eye and dry mouth. ${ }^{1}$ In addition to these sicca symptoms, patients with pSS often have multiple systemic manifestations leading to systemic damage throughout the body, including arthralgia, renal disease, pulmonary disease, and skin vasculitis. The prognosis for these patients is poor and the mortality rate is high. ${ }^{2}$ Epidemiologic data have shown that $\mathrm{pSS}$ is the second most common systemic autoimmune disease, afflicting $0.43 \%$ of the general population and is surpassed only by rheumatoid arthritis., ${ }^{3,4}$ The 
morbidity associated with pSS should not be underestimated, it commonly occurs in perimenopausal women, with a male to female ratio of $1: 10 .^{5}$

There are relatively few studies on the exposure to potential risk factors before the onset of pSS. Previous studies have suggested that, in addition to genetic influence, environmental factors also play key roles in pSS pathogenesis. For example, cigarette smoking and alcohol consumption may be negatively correlated with the risk of pSS. ${ }^{6}$ Patients with a first-degree relative (FDR) with autoimmune disease (AD) and previous pregnancies may be at increased risk for $\mathrm{pSS} .^{7}$ High psychological distress and significantly reduced quality of life are common in pSS patients, ${ }^{8,9}$ with many patients suffering from anxiety or depression. ${ }^{10}$ While some studies have described the high incidence of anxiety and depression as complications of $\mathrm{pSS},{ }^{11}$ these negative emotions will often aggravate the disease. We still do not know whether pSS is caused by or related to psychological problems before the onset of the disease.

With the acceleration of the pace of life and work pressure increasing numbers of people experience negative disorders such as anxiety and depression. It is increasingly recognized that psychological factors may trigger physical diseases. Stressful life events have been shown to play a role in several autoimmune diseases ${ }^{12,13}$ and there is a great deal of data supporting the relationship between stressful life experiences and various autoimmune diseases, including rheumatoid arthritis, ${ }^{14}$ psoriasis, and inflammatory bowel disease. ${ }^{15,16}$ There are, however, few studies relating psychological stress and pSS in clinical practice. A case-control study of pSS, lymphoma patients, and healthy participants in Greece in 2009 indicated that psychological stress and lack of social support after undergoing multiple stressful life events may be risk factors for the onset of pSS. ${ }^{17}$

A retrospective case-control study was used to select pSS patients and healthy people without psychosis, autoimmune disease, or a family history of these conditions. Smoking, alcohol use, pregnancy, menopause, and other factors were controlled at the same time to evaluate recent life stressors and stimulatory life events before the onset of pSS symptoms, and to evaluate the effects of stressors on pSS disease activity.

\section{Patients and Methods Study Design}

The study was a hospital-based case-control study in The First Affiliated Hospital of China Medical University between January and June 2019.

\section{Identification of Cases and Controls}

The cases (pSS group) were ambulatory female patients from the outpatient clinics and wards of the research hospital, who had been recently diagnosed $(<1$ year before the study) with pSS according to the 2002 International Classification of Sjogren's Syndrome. ${ }^{18}$ The inclusion criteria were age $\geq 18$ years old, no other rheumatic immune disease, no mental disease, no autoimmune disease, and no family history of mental or autoimmune disease. All patients with pSS had to be clinically stable in order to undertake the assessment. The only reason the patients had gone to the hospital was to confirm the diagnosis of pSS or to seek treatment for the management of pSS, not because of a sudden increase or change in the symptoms.

The control group (HC group) comprised healthy female participants from the physical examination center of the research hospital and who were paired to the pSS group by age \pm 3 years. The inclusion criteria were age $\geq 18$ years old, no mental disease, no autoimmune disease, and no family history of mental or autoimmune disease.

The exclusion criteria for the two groups were age $<18$ years, suffering from other autoimmune diseases, having a family history of autoimmune or mental diseases, using psychotropic drugs, and alcoholism.

The investigation lasted six months. Both groups of participants were able to read, understand, and independently complete the questionnaire.

\section{Recorded Variables and Questionnaires}

All questionnaires were assessed by specially trained investigators in the form of on-site inquiry and filling.

The basic information included age, education level, place of residence, menstrual history, pregnancy history, smoking status, and status of alcohol consumption. The clinical diagnostic and treatment history of the pSS patients was also recorded. The disease course of the patients was defined as the time from the first occurrence of dry eye, dry mouth or parotid gland enlargement to this investigation.

\section{The EULAR Sjogren's Syndrome Disease Activity Index}

The disease activity of the pSS patients was assessed by the EULAR Sjogren's syndrome disease activity index (ESSDAI) developed by the European League against Rheumatism in $2009 .{ }^{19}$ The index includes 12 domains relating to various organ systems, namely, cutaneous, respiratory, renal, articular, muscular, peripheral nervous system-related, central nervous system-related, 
hematological, glandular, constitutional, lymphadenopathic, and biological. Each domain is scored according to the severity of the symptoms experienced and the corresponding weighted indicators are assigned according to the importance of the affected system. Theoretically, the overall total score is 123 points. ${ }^{20}$

\section{The Stressful Life Events Scale}

The Stressful Life Events Scale (SLES) was modified by Desen Yang and Yalin Zhang in 1986, with reference to both the international literature and the specific characteristics of Chinese populations to describe the subjective feelings of individuals in response to stressful life events. The SLES emphasizes the individual's subjective feelings about life events and is used mostly in the fields of psychiatry and epidemiological studies of physical and mental diseases. Previous domestic studies have suggested that its Cronbach's $\alpha$ coefficient is $0.879 .{ }^{21}$ The SLES is divided into three aspects, namely, family-life, work-study, and social activity, and includes 48 common stressful life events. Each event is divided into two parts: good or bad. The influence of events was divided into five levels and the duration of influence was divided into four levels. There were three possible types of evaluation: scoring the event as a positive stimulus, scoring the event as a negative stimulus, and the total stimulus score for the event. The greater the stimulus caused by life events, the greater the mental stress.

\section{Quality Control}

The survey mainly explored the stressful life events experienced by pSS patients before the onset of the disease to explore the relationship between these and pSS onset. pSS is a chronic disease where patients may often pay no attention to its initial stages or when the diagnosis is delayed due to other causes, so the assessment needs to be certain that the stressful life events precede the onset of disease. Therefore, patients with pSS were required to fill out the SLES within one year before the manifestation of symptoms such as dry mouth, dry eyes, and parotid gland enlargement, rather than one year before the pSS diagnosis. In the HC group, the time was limited to one year before the date of questionnaire completion. In addition, to avoid recall bias, recently diagnosed cases (diagnosed within one year) were enrolled in the pSS group as far as possible.

\section{Statistical Analysis}

All data were analyzed using the SPSS statistical analysis software package (Version 23.0, IBM Corp, Armonk, NY, USA). Continuous data were expressed as mean $\pm \mathrm{SD}$ (standard deviation), and the categorical data were expressed by frequency or rate. Inter-group comparisons were calculated using the chi-square or Fisher exact tests. The associations of the number of life events (positive, negative), the score of life event stimuli (positive, negative), and the number of negative life events of different categories with pSS status were assessed as odds ratios (OR) and their respective $95 \%$ confidence interval (CI) using a univariate logistic regression model. In a multivariate logistic regression model, OR were adjusted by drinking, smoking, pregnancy history, and menopause. The Spearman rank-order correlation was used for single-factor correlation analysis. After controlling for meaningful factors, partial correlation analysis was performed on the number of negative stressful life events, the degree of stimulation of negative stressful life events, and the ESSDAI score, the correlation coefficients and significance were calculated and a scatter diagram was drawn. The statistical significance level was set at $p<0.05$.

\section{Ethics Approval and Consent to Participate}

This study was conducted in accordance with the Declaration of Helsinki and approved by the Ethics Committee of The First Affiliated Hospital of China Medical University. All participants participated voluntarily in the study and gave their written informed consent. Each participant was also informed that they had the right to refuse or to withdraw their participation at any time with no consequences attached to their participation or refusal. During the data collection, personal identifiers such as names and phone numbers of the study participants were never recorded. The collected data were kept confidential and used only for the purpose of the study.

\section{Results}

A total of 67 female pSS patients and 67 healthy controls participated in this study. There were no significant differences between the case and control groups regarding age, educational status, residential status, smoking status, family status, menopause, and pregnancy history. As shown in Table 1, the alcohol use status differed significantly between the case and control groups $(p<0.05)$. 
Table 2 shows the associations of stressful life events with pSS. Both the numbers of negative stressful life events and total numbers of stressful life events and their stimulus scores were significantly higher in pSS patients compared with controls. The associations of the negative stressful life events number $(\mathrm{OR}=2.59,95 \% \mathrm{CI}: 1.87-$ $3.58)$, total number of stressful life events $(\mathrm{OR}=2.46$, 95\% CI: 1.81-3.35), negative stressful life events stimulus scores $(\mathrm{OR}=1.31,95 \% \mathrm{CI}: 1.19-1.43)$, and pSS status remained significant in the univariate model. In contrast, the numbers of positive stressful life events and their positive stimulus scores were not significantly associated with pSS ( 1 within 95\% CI). Of the three categories of negative stressful life events, work-study stressful life events number (OR=4.83, 95\% CI: 2.48-9.40) and family-

Table I Sociodemographic Characteristics of the Case and Control Groups

\begin{tabular}{|c|c|c|c|}
\hline Variables & pSS (n=67) & $H C(n=67)$ & P-value \\
\hline Age & $55.13+7.74$ & $54.23+7.48$ & 0.695 \\
\hline Educational status & & & 0.081 \\
\hline High school and lower & $43(64.1 \%)$ & $32(47.7 \%)$ & \\
\hline College & 24 (35.9\%) & 35 (52.3\%) & \\
\hline Residential status & & & 0.214 \\
\hline Urban & 37 (55.2\%) & 45 (67.1\%) & \\
\hline Rural & $30(44.7 \%)$ & 22 (32.9\%) & \\
\hline Family status & & & 1.000 \\
\hline Single & $4(5.9 \%)$ & 5 (7.5\%) & \\
\hline Married & $63(94.1 \%)$ & 62 (92.5\%) & \\
\hline Smoking status & & & 0.485 \\
\hline Yes & $9(13.0 \%)$ & $13(19.4 \%)$ & \\
\hline No & $58(87.0 \%)$ & $54(80.6 \%)$ & \\
\hline Alcohol use status & & & $0.005^{*}$ \\
\hline Yes & $17(25.0 \%)$ & $34(50.7 \%)$ & \\
\hline No & $50(75.0 \%)$ & $33(49.3 \%)$ & \\
\hline Menopause & & & 0.387 \\
\hline Yes & $34(50.7 \%)$ & $29(43.0 \%)$ & \\
\hline No & $33(49.3 \%)$ & $38(56.8 \%)$ & \\
\hline Pregnancy history & & & 0.680 \\
\hline Yes & $63(94.1 \%)$ & 65 (97.0\%) & \\
\hline No & $4(5.9 \%)$ & $2(3.0 \%)$ & \\
\hline Treatment history & & & \\
\hline Yes & $24(35.8 \%)$ & $\mathrm{N}$ & $\mathrm{N}$ \\
\hline No & 43 (64.2\%) & $\mathrm{N}$ & $\mathrm{N}$ \\
\hline
\end{tabular}

Note: *Significant $(p<0.05)$;

Abbreviations: pSS, primary Sjogren's syndrome group; HC, healthy control group. life stressful life events number $(\mathrm{OR}=4.36,95 \% \mathrm{CI}$ : 2.58-7.28) were significantly associated with pSS, while the pSS and HC groups showed no significant differences in the stressful life events for social activity.

Table 3 shows the above results after adjustment for alcohol use status, smoking status, pregnancy history, and menopause: the number of negative stressful life events ( $\mathrm{OR}=2.56,95 \%$ CI: $1.85-3.55)$, the stimulus score for negative stressful life events $(\mathrm{OR}=1.32,95 \% \mathrm{CI}: 1.19-$ 1.46), the number of work-study-related negative stressful life events ( $\mathrm{OR}=5.51,95 \% \mathrm{CI}: 2.86-8.94)$, and the number of family-life-related negative stressful life events $(\mathrm{OR}=4.86,95 \% \mathrm{CI}: 2.50-9.49)$. All were still significantly associated with pSS.

Table 4 shows the correlation of treatment history, smoking status, alcohol consumption status, pregnancy history, menopause, and the course of the disease with the ESSDAI score. The course of the disease, smoking status, and pregnancy history were found to be significantly correlated with the ESSDAI score $(p<0.05)$, so they were used as the control factors to analyze the correlation between stressful life events and the ESSDAI score.

As shown in Table 5, the correlation between the number of negative stressful life events and the ESSDAI score was significant (partial $r=0.521, p<0.05$ ) while the correlation between the negative stressful life events stimulus score and the ESSDAI score was also significant (partial $r=0.592, p<0.05$ ).

\section{Discussion}

This study used a hospital-based retrospective case-control study design in the First Affiliated Hospital of China Medical University, a medical hub in Northeast China that has numerous outpatient and emergency departments. The survey lasted for half a year, indicating that the selected research subjects are likely to be representative of the population. As the incidence of pSS in the population is significantly higher in females than in males, and the chances of being exposed to life events depend on age, this study used female pSS patients as the research subjects and matched 67 healthy females by age \pm 3 years. There were no significant differences between the two groups of participants in terms of smoking status, menopause, and pregnancy history.

Previous studies have suggested that smoking and alcohol use may be negatively related to the risk of pSS while pregnancy history may increase the risk of developing the disease. ${ }^{22}$ In our study, the pSS group had a lower rate of 
Table 2 The Associations of Stressful Life Events with pSS

\begin{tabular}{|c|c|c|c|c|}
\hline Variables & pSS $(n=67)$ & $H C(n=67)$ & Crude OR $(95 \% \mathrm{Cl})$ & P-value \\
\hline Smoking status $(\mathrm{Y} / \mathrm{N})$ & 9/58 & $13 / 54$ & $1.55(0.6 \mathrm{I}-3.92)$ & 0.353 \\
\hline Alcohol use status $(\mathrm{Y} / \mathrm{N})$ & $17 / 50$ & $34 / 33$ & $0.33(0.16-0.68)$ & $0.030 *$ \\
\hline Menopause $(\mathrm{Y} / \mathrm{N})$ & $34 / 33$ & $29 / 38$ & $0.79(0.40-1.55)$ & 0.489 \\
\hline Pregnancy history (Y/N) & $63 / 4$ & $65 / 2$ & $2.06(0.31-11.67)$ & 0.412 \\
\hline Experiencing negative stressful life events $(\mathrm{Y} / \mathrm{N})$ & $58 / 9$ & $20 / 47$ & $15.14(6.3 \mid-36.36)$ & $0.000^{*}$ \\
\hline Experiencing positive stressful life events $(\mathrm{Y} / \mathrm{N})$ & $7 / 60$ & $3 / 64$ & $0.40(0.10-1.63)$ & 0.202 \\
\hline Number of negative stressful life events & $2.8 I \pm 1.83$ & $0.59 \pm 1.18$ & $2.59(1.87-3.58)$ & $0.000^{*}$ \\
\hline Number of positive stressful life events & $0.11 \pm 0.31$ & $0.05 \pm 0.21$ & $2.49(0.62-10.07)$ & 0.201 \\
\hline Score of negative stressful life events stimulus & $19.59 \pm|3.6|$ & $1.99 \pm 3.39$ & $1.31(1.19-1.43)$ & $0.000 *$ \\
\hline Score of positive stressful life events stimulus & $0.70 \pm 2.88$ & $0.49 \pm 2.41$ & $1.03(0.90-1.18)$ & 0.649 \\
\hline Total number of stressful life events & $2.86 \pm 1.94$ & $0.63 \pm 1.19$ & $2.46(1.81-3.35)$ & $0.000 *$ \\
\hline Total Score of stressful life events stimulus & $20.29 \pm|4.6|$ & $2.87 \pm 4.23$ & $1.23(1.16-1.36)$ & $0.000^{*}$ \\
\hline \multicolumn{5}{|l|}{ Classification of negative stressful life events } \\
\hline Number of family-life stressful life events & $1.69 \pm 1.23$ & $0.43 \pm 0.68$ & $4.36(2.58-7.28)$ & $0.000 *$ \\
\hline Number of work-study stressful life events & $0.91 \pm 1.29$ & $0.16 \pm 0.45$ & $4.83(2.48-9.40)$ & $0.01 I^{*}$ \\
\hline Number of social activity stressful life events & $0.15 \pm 0.36$ & $0.075 \pm 0.26$ & $2.18(0.70-6.75)$ & 0.178 \\
\hline Score of family-life stressful life events stimulus & $|I .4| \pm 8.83$ & $1.25 \pm 2.29$ & $1.41(1.21-1.65)$ & $0.000 *$ \\
\hline Score of work-study stressful life events stimulus & $7.46 \pm 7.80$ & $0.48 \pm 1.36$ & $1.46(1.29-1.66)$ & $0.000 *$ \\
\hline Score of social activity stressful life events stimulus & $0.73 \pm 1.90$ & $0.25 \pm 0.90$ & $1.27(0.97-1.67)$ & 0.084 \\
\hline
\end{tabular}

Note: *Significant $(p<0.05)$.

Abbreviations: $\mathrm{Y}$, yes; $\mathrm{N}$, no; $\mathrm{Cl}$, confidence interval; OR, odds ratio.

alcohol use than the control group with an OR of 0.33 (95\% CI: $0.16-0.68)$, as shown by single-factor logistic regression analysis. The lower number of alcohol drinkers among the pSS patients could be a result of oral discomfort already present before the pSS diagnosis, leading to changes in alcohol drinking habits. The smoking status was low in both groups and no significant difference was found between the two groups of participants, which may be caused by the female-only nature of the study population. However, the possibility of a protective effect of cigarette smoking should not be dismissed as it is well known that some immune-mediated chronic inflammatory diseases, such as ulcerative colitis and Behcet's disease, are less frequent among smokers and may flare up after a patient has quit smoking. ${ }^{23,24}$ Our study did not find a difference in pregnancy history between the two groups, while previous studies have suggested that women with a history of pregnancy have an increased risk of pSS. Therefore, whether pregnancy can increase the risk of pSS in women remains to be confirmed by a large-scale prospective study.

According to our findings, female pSS patients seemed to have experienced more stressful life events, particularly negative stressful events, prior to the disease onset, a finding that is consistent with Karaiskos' conclusion. ${ }^{17}$ However, the proportion of positive stressful life events was low in both the pSS and HC groups, and there was no significant difference between the two groups. This shows that, compared with positive psychological stress, negative stress is more likely to affect the body. In addition, pSS is

Table 3 Independent Correlates of pSS According to Multiple Logistic Regression Analysis

\begin{tabular}{|l|l|l|}
\hline Variables & Adjust OR (95\% Cl) & P-value \\
\hline Number of negative stressful life events & $2.56(1.85-3.55)$ & $0.000^{*}$ \\
Score of negative stressful life events stimulus & $1.32(1.19-1.46)$ & $0.000^{*}$ \\
Number of family-life stressful life events & $4.86(2.50-9.49)$ & $0.000^{*}$ \\
Number of work-study stressful life events & $5.51(2.86-8.94)$ & $0.000^{*}$ \\
Score of family-life stressful life events stimulus & $1.46(1.19-1.62)$ & $0.000^{*}$ \\
Score of work-study stressful life events stimulus & $1.39(1.27-1.68)$ & $0.000^{*}$ \\
\hline
\end{tabular}

Note: *Significant $(\mathrm{p}<0.05)$. 
Table 4 The Correlation of Pregnancy History, Menopause, Smoking Status, Alcohol Use Status, Course of Disease, and Treatment with ESSDAI Score

\begin{tabular}{|l|l|l|}
\hline \multirow{2}{*}{ Variables } & ESSDAI \\
\cline { 2 - 3 } & Correlation Coefficient & P-value \\
\hline Pregnancy history & 0.102 & 0.212 \\
Menopause & 0.132 & 0.288 \\
Smoking status & -0.304 & $0.013^{*}$ \\
Alcohol use status & -0.130 & 0.294 \\
Disease course & $0.403^{*}$ & $0.00 I^{*}$ \\
Treatment & 0.040 & 0.750 \\
\hline
\end{tabular}

Notes: *Significant $(p<0.05)$; Disease course: time from the first occurrence of dry eye, dry mouth, or parotid gland enlargement to this investigation.

a chronic disease, and its occurrence is likely to be the result of long-term accumulation of psychological stress. Froger et al described continuous or repeated stress lasting for more than 24 hours as chronic stress. ${ }^{25}$ Chronic stress can be defined as the presence of continuing and ongoing stressful circumstances, such as persistent financial difficulties or parents' divorce. Compared with acute stress, chronic stress should be the central focus of stress-related health investments. ${ }^{26}$ As a stressor, stressful life events can cause chronic stress, and can also be used as a quantitative tool for chronic stress. The relationship between stress and illness has long been recognized: Rahe and Holmes, in the 1960s, published numerous papers demonstrating the correlation and developed the Social Readjustment Rating Scale (SRRS) to quantify stress. This scale is still used in its original form by researchers conducting life-event studies over time. Stressful life events often cause elevated levels of stress and it has been found that patients that had a higher number of stressful life events reported higher perceived stress. ${ }^{27-29}$ Stress mainly involves the endocrine and nervous systems, both of which are closely linked to the immune system. Dysregulation of the hypothalamicpituitary-adrenal (HPA) axis by chronic stress has been associated with $\mathrm{pSS}^{30}$ Hypoactivation of the HPA axis as a result of chronic stress leads to reduced levels of systemic dehydroepiandrosterone-sulfate (DHEA-S). Low DHEA-S levels, in combination with disrupted intracrine processes, impair the production of the active androgen dihydrotestosterone (DHT) - which is crucial for salivary epithelial cell survival. In addition, the hypothalamic stimuli affect the anterior pituitary gland, increasing the levels of prolactin (PRL) which exerts immunomodulatory effects, ultimately leading to B-cell activating factor (BAFF) production and B-cell hyperactivation, both hallmark features of $\mathrm{pSS}^{31}$ Other studies have shown that, under chronic stress, the entire ANS system remains unbalanced and the salivary gland cells endoplasmic reticulum stress may be related to the onset of $\mathrm{pSS}^{32}$

It is thought that the human body is adaptable and disease occurs only when the body is subjected to psychological stimulation beyond its ability to adjust and adapt. Tong $\mathrm{YY}^{33}$ believed that the onset of disease due to excessive stressful mental stimulation depends on the individual's resistance to such stimulation and their ability to self-regulate, suggesting the concept of the "emotional pathogenic threshold". This study used an additional indicator, that is, the stimulus of stressful life events referring to the cumulative level of stressful life events in relation to stimulation. Our analysis indicated that the negative stressful life events stimuli score was associated with pSS, while the score for positive stressful life events stimuli was not. Besides measuring the occurrence of stressful life events, the stressful life events stimuli score is also related to the degree and duration of such events. This reflects the selfregulation ability of the human body in the face of stressful life events. With poor self-regulation, the impact of such an event is likely to be greater, especially if the duration of the stress is longer. Individual differences also play a part. For the same life event, the responses of individuals tend to be different, a complex situation that is related to many factors including early environment, family, education, and personality.

We also found that, in relation to the type of stressful life events, in both healthy people and pSS patients, the incidence of stressful life events in the family-life category was high, followed by work-study events, while the incidence of social activity stressful life events was lower. Because pSS commonly occurs in middle-aged women,

Table 5 The Correlation of Stressful Life Events and ESSDAI

\begin{tabular}{|l|l|l|l|}
\hline Control Variables & Variables & Correlation Coefficient (r) & P-value \\
\hline Smoking status & Number of negative life events & 0.521 & $0.000^{*}$ \\
Disease course & Score of negative life events stimulus & 0.592 & $0.000^{*}$ \\
\hline
\end{tabular}

Note: *Significant $(p<0.05)$. 
to maintain this demographic, we enrolled female patients aged 40 to 60 , who are more likely to suffer from familyrelated chores. It may be related to modern lifestyles that social and other life events have no obvious association with pSS. More and more people are not accustomed to participating in social activities due to work and life pressures. However, we are not sure whether social activity life events are related to pSS in male subjects, because men often require more social activities in their daily lives.

Furthermore, we also found that the number of negative stressful life events and the score of negative stressful life events stimuli were both positively correlated with the ESSDAI score. ESSDAI is an objective evaluation index, and its related examinations involving blood, serology, and pulmonary high-resolution computed tomography (HRCT) were performed after hospital admission. Fever, enlargement of lymph nodes, gland enlargement, and cutaneous lesions were determined by physical examination. The assessment of issues such as renal and muscle damage was based on the patient's medical history to determine whether further examination was required, which would prevent unnecessary invasive examination in the patients. As the patients included in this study were all in a stable condition and the ESSDAI score as a disease evaluation index is relatively fixed, the score is unlikely to change in a short time. The average ESSDAI score was 6.48, which was far lower than the highest score of 123 in the evaluation index, suggesting a low disease activity state. At the same time, we treated the index of the disease course as a covariate to eliminate possible bias between life events and ESSDAI as far as possible. Negative stressful life events may also have an impact on patients with pSS, which may aggravate the condition, and a number of clinical trials and studies have also suggested that stress can lead to changes in immune function and affect the development of certain immune-related diseases. ${ }^{22,34}$

\section{Conclusion}

In conclusion, female patients with pSS experienced more stressful life events before the onset of disease, especially negative stressful life events. The score of the negative stressful life events stimulation also has a significant impact on the development of pSS, affecting the activity of the disease in pSS patients. These results suggest that we should pay attention to the role of chronic psychological stress in the development and course of pSS in clinical practice. Paying attention to factors such as chronic stress and emotional states before the onset of pSS may be helpful in preventing the development of pSS and may provide valuable information concerning the pathogenesis and treatment of pSS.

\section{Funding}

This study was funded by the National Natural Science Foundation of China (No. 81973661).

\section{Disclosure}

The authors declare no conflicts of interest for this work.

\section{References}

1. Vivino FB. Sjogren's syndrome: clinical aspects. Clin Immunol. 2017;182:48-54. doi:10.1016/j.clim.2017.04.005

2. Voulgarelis M, Tzioufas AG, Moutsopoulos HM. Mortality in Sjögren's syndrome. Clin Exp Rheumatol. 2008;S26:66-71.

3. Helmick CG, Felson DT, Lawrence RC, et al. Estimates of the prevalence of arthritis and other rheumatic conditions in the United States. Arthritis Rheum. 2008;58(1):15-25. doi:10.1002/art.23177

4. Fox RI. Sjögren's syndrome. Lancet. 2005;366(9482):321-331. doi:10.1016/S0140-6736(05)66990-5

5. Qin BD, Wang JQ, Yang ZX, et al. Epidemiology of primary Sjögren's syndrome: a systematic review and meta-analysis. Ann Rheum Dis. 2015;74(11):1983-1989. doi:10.1136/annrheumdis2014-205375

6. Björk A, Mofors J, Wahren HM. Environmental factors in the pathogenesis of primary Sjögren's syndrome. J Intern Med. 2020;287 (5):475-492. doi:10.1111/joim.13032

7. Ben-Eli H, Aframian DJ, Ben-Chetrit E, et al. Shared medical and environmental risk factors in dry eye syndrome, Sjogren's syndrome, and B-cell non-hodgkin lymphoma: a case-control study. J Immunol Res. 2019;2019:9060842. doi:10.1155/2019/9060842

8. Bowman SJ, Booth DA, Platts RG, et al. Measurement of fatigue and discomfort in primary Sjogren's syndrome using a new questionnaire tool. Rheumatology (Oxford). 2004;43(6):758-764. doi:10.1093/rheumatology/keh170

9. Hyphantis T, Mantis D, Voulgari PV, et al. The psychological defensive profile of primary Sjögren's syndrome patients and its relationship to health-related quality of life. Clin Exp Rheumatol. 2011;29 (3):485-493.

10. Liu ZX, Hao WX. Analysis of quality of life in patients with Sjogren's syndrome. J Med Res. 2013;42:208-210.

11. Segal B, Bowman SJ, Fox PC, et al. Primary Sjögren's syndrome: health experiences and predictors of health quality among patients in the United States. Health Qual Life Outcomes. 2009;7(1):1-9. doi:10.1186/1477-7525-7-46

12. Kemeny ME, Schedlowski M. Understanding the interaction between psychosocial stress and immune-related diseases: a stepwise progression. Brain Behav Immun. 2007;21(8):1009-1018. doi:10.1016/j.bbi.2007.07.010

13. Porcelli B, Pozza A, Bizzaro N, et al. Association between stressful life events and autoimmune diseases: a systematic review and meta-analysis of retrospective case-control studies. Autoimmun Rev. 2016;15(4):325-334. doi:10.1016/j.autrev.2015.12.005

14. de Brouwer SJ, Kraaimaat FW, Sweep FC, et al. Experimental stress in inflammatory rheumatic diseases: a review of psychophysiological stress responses. Arthritis Res Ther. 2010;12(3):R89. doi:10.1186/ ar3016

15. Glaser R. Stress-associated immune dysregulation and its importance for human health: a personal history of psychoneuroimmunology. Brain Behav Immun. 2005;19(1):3-11. doi:10.1016/j.bbi.2004.06.003 
16. Song H, Fang F, Tomasson G, et al. Association of stress-related disorders with subsequent autoimmune disease. JAMA. 2018;319 (23):2388-2400. doi:10.1001/jama.2018.7028

17. Karaiskos D, Mavragani CP, Makaroni S, et al. Stress, coping strategies and social support in patients with primary Sjogren's syndrome prior to disease onset: a retrospective case-control study. Ann Rheum Dis. 2009;68(1):40-46. doi:10.1136/ard.2007.084152

18. Vitali C, Bombardieri S, Jonsson R, et al. Classification criteria for Sjögren's syndrome: a revised version of the European criteria proposed by the American-European consensus group. Ann Rheum Dis. 2002;61(6):554-558. doi:10.1136/ard.61.6.554

19. Seror R, Ravaud P, Bowman SJ, et al. EULAR Sjögren's syndrome disease activity index: development of a consensus systemic disease activity index for primary Sjogren's syndrome. Ann Rheum Dis. 2010;69(6):1103-1109. doi:10.1136/ard.2009.110619

20. Seror R, Bowman SJ, Brito-Zeron P, et al. EULAR Sjogren's syndrome disease activity index (ESSDAI): a user guide. RMD Open. 2015;1(1):e000022. doi:10.1136/rmdopen-2014-000022

21. Wang Y, Liang CG. Relationship among character strength, life stress events and emotional well-being in nursing staffs. Chin Occup Med. 2017;44:610-614.

22. Yang EV, Glaser R. Glaser R: stress-induced immunomodulation and the implications for health. Int Immuopharmacol. 2002;2(2-3):315-324. doi:10.1016/S1567-5769(01)00182-5

23. Birrenbach T, Böcker U. Inflammatory bowel disease and smoking: a review of epidemiology, pathophysiology, and therapeutic implications. Inflamm Bowel Dis. 2004;10(6):848-859. doi:10.1097/ 00054725-200411000-00019

24. Soy M, Erken E, Konca K, et al. Smoking and behçet's disease. Clin Rheumatol. 2000;19(6):508-509. doi:10.1007/s100670070020

25. Froger N, Palazzo E, Boni C, et al. Neurochemical and behavioral alterations in glucocorticoid receptor-impaired transgenic mice after chronic mild stress. J Neurosci. 2004;24(11):2787-2796. doi:10.1523/ JNEUROSCI.4132-03.2004
26. Lantz PM, House JS, Mero RP, et al. Stress, life events, and socioeconomic disparities in health: results from the Americans' changing lives study. J Health Soc Behav. 2005;46(3):274-288. doi:10.1177/ 002214650504600305

27. Paluch AE, Shook RP, Hand GA, et al. The influence of life events and psychological stress on objectively measured physical activity: a 12-month longitudinal study. J Phys Act Health. 2018;15(5):374-382. doi:10.1123/jpah.2017-0304

28. Pancar YE, Durmus D, Sarisoy G. Perceived stress, life events, fatigue and temperament in patients with psoriasis. J Int Med Res. 2019;47(9):4284-4291. doi:10.1177/0300060519862658

29. Aas M, Vecchio C, Pauls A, et al. Biological stress response in women at risk of postpartum psychosis: the role of life events and inflammation. Psychoneuroendocrinology. 2020;113:104558. doi:10.1016/j.psyneuen.2019.104558

30. Johnson EO, Vlachoyiannopoulos PG, Skopouli FN, et al. Hypofunction of the stress axis in Sjögren's syndrome. J Rheumatol. 1998;25(8):1508-1514.

31. Mavragani CP, Fragoulis GE, Moutsopoulos HM. Endocrine alterations in primary Sjogren's syndrome: an overview. J Autoimmun. 2012;39(4):354-358. doi:10.1016/j.jaut.2012.05.011

32. Skopouli FN, Katsiougiannis S. How stress contributes to autoimmunity-lessons from Sjögren's syndrome. FEBS Lett. 2018;592(1):5-14. doi:10.1002/1873-3468.12933

33. Tong YY. Analysis of the connotation of the mechanism of seven emotions causing diseases. J Trad Anhui Chin Med Coll. 1996;2:4-7.

34. Moynihan JA. Mechanisms of stress-induced modulation of immunity. Brain Behav Immun. 2003;17(1):S11-6. doi:10.1016/ S0889-1591(02)00060-0
Neuropsychiatric Disease and Treatment

\section{Publish your work in this journal}

Neuropsychiatric Disease and Treatment is an international, peerreviewed journal of clinical therapeutics and pharmacology focusing on concise rapid reporting of clinical or pre-clinical studies on a range of neuropsychiatric and neurological disorders. This journal is indexed on PubMed Central, the 'PsycINFO' database and CAS, and

\section{Dovepress}

is the official journal of The International Neuropsychiatric Association (INA). The manuscript management system is completely online and includes a very quick and fair peer-review system which is all easy to use. Visit http://www.dovepress.com/testimonials.php to read real quotes from published authors. 